\title{
Preparation for open heavy flavour measurements with the ALICE detector at the LHC
}

\section{Andrea Rossi*}

University of Padova \& INFN

E-mail: rossialpd.infn.it

\section{for the ALICE Collaboration}

The ALICE experiment has started collecting proton-proton collision data at the CERN Large Hadron Collider (LHC) and will collect the first $\mathrm{Pb}-\mathrm{Pb}$ data in autumn 2010. Heavy quarks are regarded as powerful tools to investigate the properties of QCD matter at the extreme conditions reached in collisions between nuclei. We review the ALICE open heavy-flavour program and show that the ALICE detector is ready to accomplish it.

XVIII International Workshop on Deep-Inelastic Scattering and Related Subjects April 19 -23, 2010

Convitto della Calza, Firenze, Italy

\footnotetext{
* Speaker.
} 


\section{Introduction: heavy quarks at the LHC}

ALICE [1], 2] is the LHC experiment dedicated to the study of heavy-ion collisions. $\mathrm{Pb}-\mathrm{Pb}$ collisions at a centre of mass energy per nucleon pair $\sqrt{s_{\mathrm{NN}}}=2.75 \mathrm{TeV}$ are scheduled for the end of 2010. The measurement of charm and beauty hadrons will allow to investigate the mechanisms of heavy-quark production, propagation and hadronization in the hot and dense QCD medium formed in these collisions. High energy partons, produced in the first stage of a collision in hardscattering processes with high momentum transfer, are expected to lose energy while traversing the medium. The study of the energy loss mechanism and of its dependence on medium properties, like the energy density, can provide information on the medium itself. Due to the QCD nature of parton energy loss, quarks are predicted to lose less energy than gluons because of the different colour charge (Casimir factor). In addition, the dead-cone effect is expected to reduce the energy loss of massive quarks with respect to light partons [B]. This scenario can be tested, from an experimental point of view, by comparing the nuclear modification factors of light hadrons $\left(R_{\mathrm{AA}}^{h}\right)$, mostly originating from gluon fragmentation, with that of hadrons with charm $\left(R_{\mathrm{AA}}^{\mathrm{D}}\right)$ or beauty $\left(R_{\mathrm{AA}}^{\mathrm{B}}\right)^{1}$. A pattern $R_{\mathrm{AA}}^{h}\left(p_{\mathrm{t}}\right) \lesssim R_{\mathrm{AA}}^{\mathrm{D}}\left(p_{\mathrm{t}}\right) \lesssim R_{\mathrm{AA}}^{\mathrm{B}}\left(p_{\mathrm{t}}\right)$ has been predicted [ $\llbracket$ ] .

Information on the medium temperature can be provided by measuring the yields of charmonium and bottomonium states. Quarkonium states are sensitive to the initial temperature of the system due to colour screening that can prevent their formation. In this proceeding we will focus on open charm and beauty measurements. More information on ALICE capability to detect charmonium and bottomonium states can be found in [2].

Heavy-quark production measurements in pp collisions at the LHC, besides providing a necessary reference for the study of medium effects in $\mathrm{Pb}-\mathrm{Pb}$ collisions, are interesting per se, as a test of perturbative QCD (pQCD) in a new domain, up to 7 times above the present energy frontier at the Tevatron.

The $c \bar{c}$ and $b \bar{b}$ production yields per event assumed as a baseline for the ALICE simulation studies are: $N_{\mathrm{tot}}^{\mathrm{cc}[\mathrm{b} \bar{b}]}=0.10[0.003]$ and $N_{\mathrm{tot}}^{\mathrm{cec}[\mathrm{b} \bar{b}]}=0.16[0.006]$ for pp collisions at $\sqrt{s}=7 \mathrm{TeV}$ and $\sqrt{s}=$ $14 \mathrm{TeV}$ respectively; $N_{\mathrm{tot}}^{\mathrm{cc}[\mathrm{b} \bar{b}]}=56[2]$ and $N_{\mathrm{tot}}^{\mathrm{c} \overline{\mathrm{c}}[\mathrm{b} \bar{b}]}=115[4.6]$ for the most $5 \%$ central $\mathrm{Pb}-\mathrm{Pb}$ collisions at $\sqrt{s_{\mathrm{NN}}}=2.75 \mathrm{TeV}$ and $\sqrt{s_{\mathrm{NN}}}=5.5 \mathrm{TeV}$ respectively. These numbers are obtained from pQCD calculations at NLO [5] with a reasonable set of parameters [2], including nuclear shadowing.

\section{Heavy flavour measurements in progress}

At LHC energies $2000 \div 3000$ charged particles per unit of rapidity might be produced in a central $\mathrm{Pb}-\mathrm{Pb}$ collision. The ALICE capability to detect open charm and beauty hadrons in the central barrel in this environment is provided by:

- Tracking system: the Time Projection Chamber (TPC), the Inner Tracking System (ITS) and the Transition Radiation Detector (TRD), embedded in a magnetic field of $0.5 \mathrm{~T}$, allow for track reconstruction in the pseudorapidity range $|\eta|<0.9$, with a $p_{\mathrm{t}}$ resolution better than

\footnotetext{
${ }^{1}$ The nuclear modification factor, defined as $R_{\mathrm{AA}}\left(p_{\mathrm{t}}\right)=\frac{\mathrm{d} N_{\mathrm{AA}} / \mathrm{d} p_{\mathrm{t}}}{\left\langle N_{\text {coll }}\right\rangle N_{p p} / \mathrm{d} p_{\mathrm{t}}}$, is the ratio between the measured yield of a given particle/set of particles in AA collisions $\left(N_{\mathrm{AA}}\right)$ and the correspondent yield in pp collisions $\left(N_{p p}\right)$ scaled by the number of binary collisions $\left\langle N_{\text {coll }}\right\rangle$ to obtained the yield expected in AA collisions in the absence of medium effects.
} 
$2 \%$ up to $20 \mathrm{GeV} / c$ and a transverse impact parameter resolution better than $60 \mu \mathrm{m}$ for $p_{\mathrm{t}}>1 \mathrm{GeV} / c$. The ITS is composed of six layers of silicon detectors: the two outermost layers are strips (SSD), in the middle there are two layers of drift detectors (SDD) and the two innermost are pixel (SPD). The latter (inner radius $\sim 3.9 \mathrm{~cm}$ ) is the main detector for the reconstruction of the primary vertex of interaction and of secondary decay vertices.

- Particle IDentification (PID) system: the measurement of the energy loss $(\mathrm{d} E / \mathrm{d} x)$ in the TPC and in the ITS and of the time-of-flight in the TOF detector allow a good separation among kaons, protons and pions up to $\sim 2.5 \mathrm{GeV} / c$. The separation of electrons from charged hadrons is achieved with the TRD, with the TPC and with the electromagnetic calorimeter (EMCAL).

The forward Muon Spectrometer, covering a pseudorapidity region $-4<\eta<-2.5$, is used for track reconstruction as well as for muon identification. The detector is composed of five tracking stations with two planes of Multi-Wire Proportional Chambers each, having a spatial resolution of about $100 \mu \mathrm{m}$, a dipole magnet with an integral field of $3 \mathrm{~T} \cdot \mathrm{m}$ and two trigger stations of Resistive Plate Chambers placed behind an iron-wall muon filter with a thickness of about 7 interaction lengths. A front absorber is placed in front of the spectrometer, $90 \mathrm{~cm}$ from the collision point, to absorb hadrons and photons from the interaction vertex.

Simulation studies [2] indicate that ALICE will be able to measure charm and beauty production in $\mathrm{Pb}-\mathrm{Pb}$ as well as in proton-proton collisions, exploiting its tracking and particle identification systems. The main analyses in preparation are:

- Open charm. Exclusive reconstruction of charm hadron decays into hadrons, $\mathrm{D}^{0} \rightarrow \mathrm{K}^{-} \pi^{+}$, $\mathrm{D}^{+} \rightarrow \mathrm{K}^{-} \pi^{+} \pi^{-}, \mathrm{D}^{\star+} \rightarrow \mathrm{D}^{0} \pi_{\text {soft }}^{+}, \mathrm{D}^{0} \rightarrow \mathrm{K}^{-} \pi^{+} \pi^{-} \pi^{+}, \mathrm{D}_{s}^{+} \rightarrow \mathrm{K}^{-} \mathrm{K}^{+} \pi^{+}, \Lambda_{c}^{+} \rightarrow p \mathrm{~K}^{-} \pi^{+}$(under study) in $|\eta|<0.9$; single muons and di-muons in $-4<\eta<-2.5$.

- Open beauty. Inclusive single leptons, $\mathrm{B} \rightarrow \mathrm{e}+X$ in $|\eta|<0.9$ and $\mathrm{B} \rightarrow \mu+X$ in $-4<\eta<$ -2.5 ; inclusive displaced charmonia $\mathrm{B} \rightarrow \mathrm{J} / \Psi\left(\rightarrow \mathrm{e}^{+} \mathrm{e}^{-}\right)+X$ in $|\eta|<0.9 ; b$-tagging of jets reconstructed in the tracking detectors and in the EMCAL (under study).

\subsection{Detector performance after the first proton-proton collisions at $7 \mathrm{TeV}$}

The possibility to carry out this rich heavy-flavour programme is strongly related to ALICE capability in reconstructing secondary vertices displaced from the primary vertex. The required "resolving power" is dictated by the typical impact parameter of the tracks produced by the decay of charm and beauty hadrons. For instance, the typical impact parameter of the kaon and pion tracks coming from the decay of a $\mathrm{D}^{0}$ is of the order of $\mathrm{c} \tau\left(\mathrm{D}^{0}\right) \approx 124 \mu \mathrm{m}$. The impact parameter resolution is determined mainly by the ITS detector, especially by the SPD. Therefore, the alignment of the ITS and especially of the SPD is a key point to guarantee the spatial precision needed for the reconstruction of primary and secondary vertices. The alignment strategy and the results achieved with cosmic data collected in 2008 are described in [5]. About $80 \%$ of the SPD modules were aligned to a level such that the worsening of spatial resolution due to misalignment was kept within $20 \%$ of the ideal resolution. The survey measurements performed during SSD assembly and mounting phases assured a satisfactory first-alignment status for this detector. Tracks from 
proton-proton collisions are now used to align the SPD modules on the sides (poorly illuminated by cosmic-rays) and to monitor and improve the alignment quality of the whole ITS. The current residual misalignment is estimated to be $\sim 7 \mu \mathrm{m}$ for the SPD and $\lesssim 10 \mu \mathrm{m}$ for the SSD. For the SDD, to account for possible miscalibrations of the minimum drift time and of the drift velocity, alignment and calibration parameters are recovered together module-by-module: the current "effective" resolution has been evaluated to be $\sim 65 \mu \mathrm{m}$.
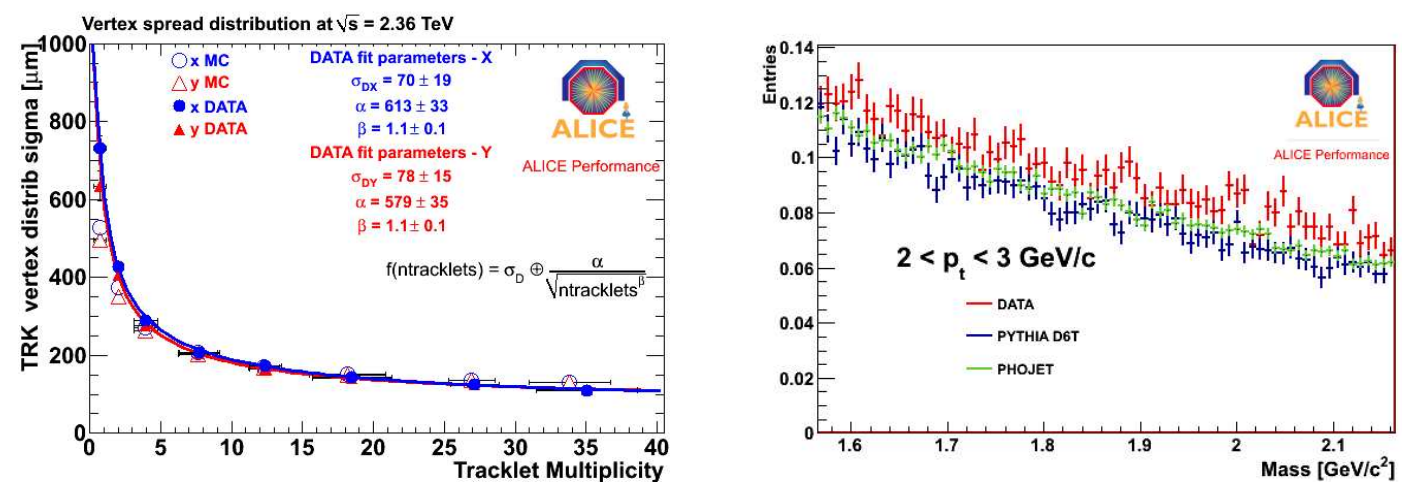

Figure 1: On the left: spread of $x$ and $y$ coordinates of the reconstructed primary vertex for pp collisions at 2.36 TeV for data (filled markers) and MC (empty markers). The formula reported in the panel is used to fit data points (continuous lines). On the right: invariant mass distribution observed with $\sim 3.7 \cdot 10^{5}$ events from pp collisions at $\sqrt{s}=900 \mathrm{GeV}$ for $2<p_{\mathrm{t}}^{\mathrm{D}^{0}}<3 \mathrm{GeV} / c$. MC distributions obtained by using two different event generators (PYTHIA D6T and PHOJET) are also shown (the distributions are scaled to the same number of events).

The $z$ coordinate (along the beam line) of the interaction point is distributed in a range of several centimetres. The beam spot size in the transverse plane is of the order of tenths-hundreds micron, depending on the beam optics $\left(\beta^{\star}\right)$ and energy. Two algorithms 汤 are used to reconstruct the primary vertex position. The first exploits the correlation between pairs of points in the SPD ("tracklets"). The efficiency reaches $\sim 90 \%$ with a tracklet multiplicity of 4 and approaches $100 \%$ when the tracklet multiplicity is 8 . If the calculation of the full 3D position fails, the $z$ coordinate alone is calculated: in this case one tracklet is sufficient to get $100 \%$ efficiency. The second algorithm is based on the straight line approximation of full reconstructed tracks in the vicinity of the vertex. With a tracklet multiplicity of 3 , the reconstruction efficiency is $\sim 95 \%$ if the information of the luminous region, position and size, is used to constrain the vertex, $\sim 80 \%$ otherwise; $100 \%$ is approached at higher multiplicity. In Fig. 1 (left panel) the spread of the reconstructed $x$ and $y$ coordinates as a function of the tracklet multiplicity of the event is reported. Monte Carlo points are in good agreement with data. Data points are fitted with the function $\sigma_{D} \oplus \frac{\alpha}{\sqrt{N_{t r k l}^{\beta}}} . \sigma_{D}$ describes the asymptotic value of the curve, determined by the size of the luminous region along the considered axis, while the second term represents a parametrisation of the primary vertex resolution as a function of the tracklet multiplicity.

\subsection{Charm and beauty measurements at central rapidity}

The $\mathrm{D}^{0} \rightarrow \mathrm{K}^{-} \pi^{+}$(branching ratio $\approx 3.8 \%$ ) is among the most promising channels for open 
charm detection. The detection strategy is based on an invariant mass analysis of all the pairs of tracks with opposite charges. To cope with the large combinatorial background, displaced-vertex topologies are looked for, i.e. tracks displaced from the primary vertex are selected and good alignment between the reconstructed meson momentum and its flight direction is required. The accessible $p_{\mathrm{t}}$ range for the $\mathrm{D}^{0}$ is $1-20 \mathrm{GeV} / c$ for $10^{7}$ central $\mathrm{Pb}-\mathrm{Pb}$ events at $\sqrt{s_{\mathrm{NN}}}=5.5 \mathrm{TeV}$ and $0.5-20 \mathrm{GeV} / c$ for $10^{9} \mathrm{pp}$ events at $\sqrt{s}=14 \mathrm{TeV}$, with statistical errors better than $15-20 \%$ at high $p_{\mathrm{t}}$. Similar performances are expected also for the $\mathrm{D}^{+} \rightarrow \mathrm{K}^{-} \pi^{+} \pi^{-}$channel $(\mathrm{c} \tau \approx 300 \mu \mathrm{m}$, branching ratio $\approx 9.2 \%$ ). Systematic errors (acceptance and efficiency corrections, centrality selection for $\mathrm{Pb}-\mathrm{Pb}$ ) are expected to be smaller than $20 \%$. In Fig. 1 (right panel) the invariant mass distribution observed with $\sim 3.7 \cdot 10^{5}$ events from pp collisions at $\sqrt{s}=900 \mathrm{GeV}$ is shown for $2<p_{\mathrm{t}}^{\mathrm{D}^{0}}<3 \mathrm{GeV} / c$. No signal peak is expected with these statistics. MC distributions obtained by using two different event generators are also shown. About two weeks after this conference a first peak with significance $\sim 5$ was observed for $p_{\mathrm{t}}^{\mathrm{D}^{0}}>2 \mathrm{GeV} / c$ with $2.4 \cdot 10^{7}$ pp events at $\sqrt{s_{\mathrm{NN}}}=7 \mathrm{TeV}$.

The production of open beauty at central rapidity, $|y|<1$, can be studied by detecting the semielectronic decays of $\mathrm{b}$-hadrons (branching ratio $\approx 10 \%$ ). A high-purity sample of electrons from beauty can be obtained exploiting the electron identification capability of the TRD and the TPC and selecting tracks displaced from the primary vertex (the average impact parameter of electrons from beauty is $d_{0} \approx 500 \mu \mathrm{m}$ ): a cut on the impact parameter reduces the semi-electronic charm-decay background component, rejects misidentified $\pi^{ \pm}$and $\mathrm{e}^{ \pm}$from Dalitz decays and $\gamma$ conversions. As an example, with $10^{7}$ central $\mathrm{Pb}-\mathrm{Pb}$ events at $\sqrt{s_{\mathrm{NN}}}=5.5 \mathrm{TeV}$ and for $p_{\mathrm{t}}>2 \mathrm{GeV} / c$, a raw signal of $\sim 8 \cdot 10^{4}$ with a purity $\sim 80 \%$ is expected if a $p_{\mathrm{t}}$ dependent $200<d_{0}<600 \mu \mathrm{m}$ cut is applied. This will allow the measurement of electron-level $p_{\mathrm{t}}$-differential cross-section in the range $2<p_{\mathrm{t}}<20 \mathrm{GeV} / c$ with a statistical error smaller than $15 \%$ at high $p_{\mathrm{t}}$. A similar performance is expected for pp collisions.

\subsection{Charm and beauty measurements at forward rapidity}

Charm and beauty production can be measured also in the forward muon spectrometer, in the pseudorapidity range $-4<\eta<-2.5$, by analyzing the single-muon and di-muon invariant mass distributions [2]. The main background to the 'heavy-flavour muon signal' are $\pi^{ \pm}$and $\mathrm{K}^{ \pm}$decays. A cut at $p_{\mathrm{t}}>1.5 \mathrm{GeV} / c$ is applied to all reconstructed muons in order to increase the signal-tobackground ratio. Then, a fit technique allows to extract a $p_{\mathrm{t}}$ distribution of muons from $\mathrm{B}$ decays. Since only minimal cuts are applied, statistical errors are expected to be smaller than $5 \%$ up to a muon $p_{\mathrm{t}} \approx 30 \mathrm{GeV} / c$. Systematic errors, mainly due to the assumptions in the fit procedure, are expected to be lower than $20 \%$.

\section{Expected sensitivity for the study of the energy loss in the medium}

By comparing to theoretical predictions the expected ALICE precision for the measurement of the nuclear modification factors $R_{\mathrm{AA}}^{\mathrm{D}}$ and $R_{\mathrm{AA}}^{\mathrm{B}}$, and for the heavy-to-light ratio $R_{\mathrm{D} / h}$ (ratio between $R_{\mathrm{AA}}^{\mathrm{D}}$ and $R_{\mathrm{AA}}^{h}$ ) and $R_{\mathrm{AA}}^{e}$ from B $/ R_{\mathrm{AA}}^{e}$ from D, it has been shown [8] that the charm and beauty measurements described above can be used to test the expected colour-charge and mass dependence of 

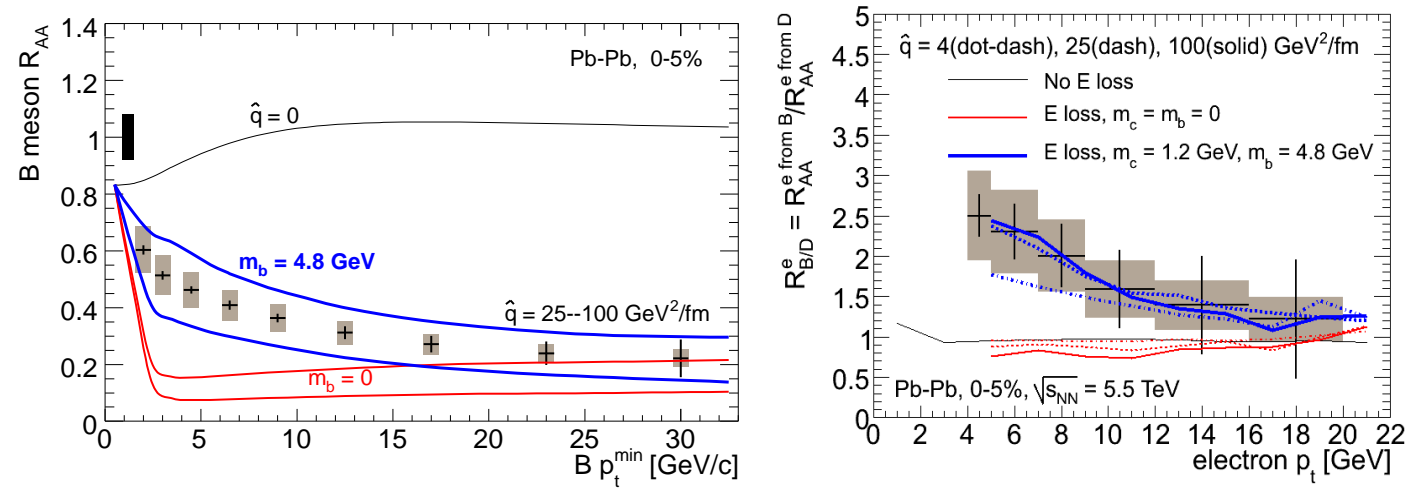

Figure 2: Expected performance for the nuclear modification factor of B mesons (left) and for the ratio of the nuclear modification factors of B-decay electrons and of D-decay electrons (right). Predictions [ [4] with and without the effect of the heavy-quark mass, for a medium transport coefficient $\hat{q}$ (a measurement of the medium density) in the range $25-100 \mathrm{GeV}^{2} / \mathrm{fm}$ are shown. Errors, corresponding to the centre of the prediction bands for massive quarks, are: bars=statistical, shaded area=systematic.

parton energy loss in the medium. The sensitivity to $R_{\mathrm{AA}}^{\mathrm{B}}$ and $R_{\mathrm{AA}}^{e \text { from B }} / R_{\mathrm{AA}}^{e \text { from D }}$ is presented in Fig. 2.

\section{Summary}

The abundant production of heavy quarks at LHC energies will allow to address several issues of heavy-ion physics and of QCD under extreme conditions: from the predictions of $\mathrm{pQCD}$ at a new energy scale, in pp collisions, to the mechanism of energy loss in a QCD medium, in $\mathrm{Pb}-\mathrm{Pb}$. The commissioning of the ALICE detector with cosmic-rays is being refined with pp collision data at different centre-of-mass energies: the tracking, vertexing and particle identification performance is already close to that by design, required to accomplish ALICE rich heavy-flavour physics program.

\section{References}

[1] F. Carminati et al. [ALICE Collaboration], J. Phys. G: Nucl. Part. Phys. 30 (2004) 1517.

[2] B. Alessandro et al. [ALICE Collaboration], J. Phys. G: Nucl. Part. Phys. 32 (2006) 1295.

[3] Y.L. Dokshitzer and D.E. Kharzeev, Phys. Lett. B 519 (2001) 199.

[4] N. Armesto, A. Dainese, C.A. Salgado and U.A. Wiedemann, Phys. Rev. D 71 (2005) 054027.

[5] M. Mangano, P. Nason and G. Ridolfi, Nucl. Phys. B 373 (1992) 295.

[6] ALICE collaboration 2010 JINST 5 P03003.

[7] E. Bruna et al., ALICE Internal Note 2009-018 (2009).

[8] A. Dainese [ALICE Collaboration], J. Phys. G 35 (2008) 044046; Nucl. Phys. A 783 (2007) 417. 